Classification

Physics Abstracts

$61.16 \mathrm{~B}-61.16 \mathrm{C}-82.65 \mathrm{~J}$

\title{
Model catalysts obtained by cluster deposition of Palladium onto HOPG. TEM and STM characterisation
}

\author{
Francisco José Cadete Santos Aires $\left({ }^{1}\right)$, Philippe Sautet $\left({ }^{1}\right)$, Gilbert Fuchs $\left({ }^{2}\right)$, Jean-Luc \\ Rousset $\left({ }^{1}\right)$ and Patrice Mélinon $\left({ }^{2}\right)$ \\ (1) Institut de Recherches sur la Catalyse (CNRS) 2, Avenue Albert Einstein, 69626 Villeurbanne \\ cedex, France \\ ( $\left.{ }^{2}\right)$ Département de Physique des Matériaux (UCB Lyon I) 43, Boulevard du 11 Novembre 1918, \\ 69622 Villeurbanne cedex, France
}

(Received August 20, 1993; accepted October 1993)

\begin{abstract}
Résumé. - Des particules supportées (catalyseurs modèles utilisés dans des réactions d'hydrogénation) sont obtenues par déposition de jets d'agrégats sur HOPG et sont caractérisées par microscopie électronique à transmission (TEM) et par microscopie à effet tunnel (STM). Les observations en TEM montrent la formation de groupes de particules sur la surface et la décoration des marches du graphite. Les groupes de particules sur la surface sont bien séparés entre eux. Les observations STM à grande échelle sont en accord avec les observations de TEM. Une étude expérimentale détaillée des modulations périodiques $(\sqrt{3} \times \sqrt{3}) \mathrm{R} 30^{\circ}$ de la densité de charge du graphite observées autour des particules est présentée et ces modulations sont interprétées en tant qu'une perturbation introduite par la particule au niveau des fonctions d'onde dégénérées du graphite, levant la dégénérescence et donnant lieu à la superstructure $(\sqrt{3} \times \sqrt{3}) \mathrm{R} 30^{\circ}$.
\end{abstract}

\begin{abstract}
Supported particles (model catalysts used in hydrogenation reactions) are obtained by cluster beam deposition onto HOPG and characterised by transmission electron microscopy (TEM) and scanning tunneling microscopy (STM). TEM shows the formation of groups of particles on the surface and the decoration of the steps of graphite. The groups of particles on the surface are well separated from each other. STM large scale observations agree with these observations. A detailed experimental study of the $(\sqrt{3} \times \sqrt{3}) \mathrm{R} 30^{\circ}$ periodic charge density modulations of the graphite observed around the particles is presented and these modulations are interpreted in terms of a perturbation introduced by the particle to the degenerate wave functions of graphite, lifting up the degeneracy and yielding the $(\sqrt{3} \times \sqrt{3}) \mathrm{R} 30^{\circ}$ superstructure.
\end{abstract}

\section{Introduction.}

The characterisation of supported metal particles, known as model catalysts used in hydrogenation reactions, is of great interest in order to understand their catalytic properties and as a reference to the study of more realistic catalysts. It is well established that the physical characteristics of these supported particles (particles size, distribution and shape, valence state, electronic structure, their 
interaction with the support, ...) are intimately bound to their catalytic properties such as the activity and the selectivity.

Transmission electron microscopy (TEM) is currently used to characterise these collections of particles and several authors have shown the interest of using scanning tunnelling microscopy (STM) to probe the surface of the particles at the atomic scale (with access to the third dimension) [1 - 4], to study the charge density modulations of the graphite around the particles, which is a sign of their interaction with the substrate $[3,5]$, or to try to observe any difference between particles before and after reaction $[4,6]$. No changes in morphology were observed for palladium particles observed by STM in air and in UHV [2].

Generally, these supported particles are generated by atomic vapour deposition onto supposedly inert substrates, such as highly oriented pyrolitic graphite (HOPG), in order to try to preserve the intrinsic properties of the metallic particles.

We report here a study on palladium supported particles HOPG obtained by low-energy cluster beam deposition from a laser vaporisation generator [7]. The choice of this method has to do with the fact that we can obtain well controlled size distributions of free clusters that after deposition have proven to form different and better controlled supported particles distributions than those obtained by atomic or molecular deposition, in the case of other metals [8 - 10]. In addition, LECBD technique allows the study of the coverage rate independently of the cluster size.

TEM and STM are used to characterise the obtained particles and special interest is taken on the interpretation of the charge density modulations of the graphite observed by STM around the palladium particles.

\section{Experimental procedure.}

2.1 CluSTER DEPOSITION. - The generator used to obtain the cluster beam is the so-called laser vaporisation generator [7]. The principle is to use a Nd: YAG laser to vaporise the metal from a rod to form a plasma and to introduce (in a pulsed mode) an inert gas (helium) that will induce the nucleation and growth of the clusters. The mean size of the clusters can be monitored by changing the inert gas pressure and the residence time in the nucleation cell. In order to avoid the laser to touch the metallic rod always on the same spot, the rod rotates and translates vertically. The free clusters are then extracted through a skimmer, by differential pumping, and form a cluster beam which carries neutral and ionised clusters. The size distribution of the free ionised clusters is then characterised by time of flight mass spectrometry (TOFMS). By controlling the rate of deposition, with a vibrating quartz, before and after removal of the ionised clusters (by magnetic deflection) two modes of cluster growth can be controlled. Firstly, the ionised clusters produced by the generator (anions and cations) can be analysed. Secondly, the neutral species can be photoionised by a $\mathrm{XeCl}$ excimer pumping a dye laser and analysed. In several materials, we have checked that the mass abundance features were roughly the same for both neutral and ionised clusters. After removal of the ionised clusters, we can then deposit the neutral ones onto the HOPG (cleaved and annealed) substrates. Deposition was performed at room temperature $(\approx$ $300 \mathrm{~K}$ ) under a background pressure of roughly $10^{-4} \mathrm{~Pa}$. The clusters are reasonnably supposed to be thermalised by the helium gas $\left(\approx 1500 \mathrm{~ms}^{-1}\right)$ and so their kinetic energy (a few tenth of $\mathrm{eV} /$ atom) is much smaller than the binding energy in metals (a few eV/atom). So these low-energy clusters do not dissociate when the impact with the substrate is produced.

Free clusters grow by accretion process. Clusters such as $\mathrm{Pd}_{3}^{+}$are unstable and do not allow the cluster growth. For palladium, only negatively charged clusters exist and are observe by TOFMS. We have deposited a cluster distribution centred around 50 atoms $\left(\operatorname{Pd}_{50}\right)$ and with a size dispersion of 50. There are no traces of monomers. The deposited amount of palladium, monitored by a vibrating quartz, corresponds to $3 \times 10^{15}$ atoms $/ \mathrm{cm}^{2}$. 
2.2 CHARACTERISATION OF THE SUPPORTED PARTICLES. - After air transfer, two samples obtained in the same experimental conditions were studied by TEM and STM.

After cleaving one of the HOPG crystals containing the particles, TEM in a JEOL 200CX electron microscope, operating at $200 \mathrm{kV}$, was performed to study the collections of supported particles. Image analysis of the obtained micrographs was used to obtain the mean size of the particles and their density on the substrate.

For STM the sample did not need any further preparation and the experiment was run in a Nanoscope II of Digital Instruments. The images were obtained with a bias voltage of $50 \mathrm{mV}$ and a tunnel current of $2 \mathrm{nA}$. The tip was obtained by cutting (diagonal cutters) a PtIr (80/20) wire. Large scale images were obtained on the topographic mode (constant current), while high spatial resolution images were obtained in the current mode (constant height).

\section{TEM results.}

Figure 1 shows two electron micrographs corresponding to the Pd/HOPG sample. Two main characteristics can be seen:

i) Several rows of particles decorate the graphite steps (Fig. 1b, on the left).

ii) On the surface of HOPG, particles arrange themselves in large groups that can be formed of 3 up to several tens of particle (Figs. 1a and b). These groups are well separated from each other (20 to $200 \mathrm{~nm})$.

These two observations and the parameters deduced from the analysis of the micrographs generate several comments. The arrangement of the particles in groups is quite peculiar since, generally (in atomic deposition), particles are evenly distributed on the surface [11]. One reason can be advanced to explain this peculiarity. While the HOPG samples in other studies [11] were annealed in-situ, our HOPG samples were air cleaved, then annealed in a separate chamber and then air transferred to the cluster generator. This implies the presence at the surface of our samples of adsorbates and impurities that act as nucleation sites. In the case of $i$-situ cleaved HOPG all nucleation sites will then appear as equivalent and randomly distributed, while in our case the impurities generate preferential nucleation sites to the incoming clusters. However, we cannot eliminate the hypothesis of a particular behaviour of the nucleation and growth of the incoming clusters (due to their peculiar properties somewhere between the atom and the bulk) by comparison to the atomic condensation, since the same kind of behaviour has been observed with antimony [12], for instance.

Image analysis of the micrographs gave us a quantification of the parameters of the collection of particles on the surface. The mean size of the particles is $D=3.8 \mathrm{~nm}$ and their density on the substrate is $N=4.8 \times 10^{11}$ particles $/ \mathrm{cm}^{2}$. By comparison to a similar sample obtained by atomic deposition $\left(D=2.8 \mathrm{~nm}\right.$ and $N=6 \times 10^{12}$ particles $\left./ \mathrm{cm}^{2}\right)$ [11], we have larger particles and a weaker density; but the parameter which is worth noting is that $D$ is about four times larger than the diameter of an incoming free cluster $(1 \mathrm{~nm})$ which means that each (medium size) particle is formed by about 60 incoming clusters. This is an important indication that the incoming clusters are mobile on the substrate. They do not stay still on the substrate and do not grow only by the incoming flux of clusters. They diffuse, nucleate and grow as if they were large atoms. 


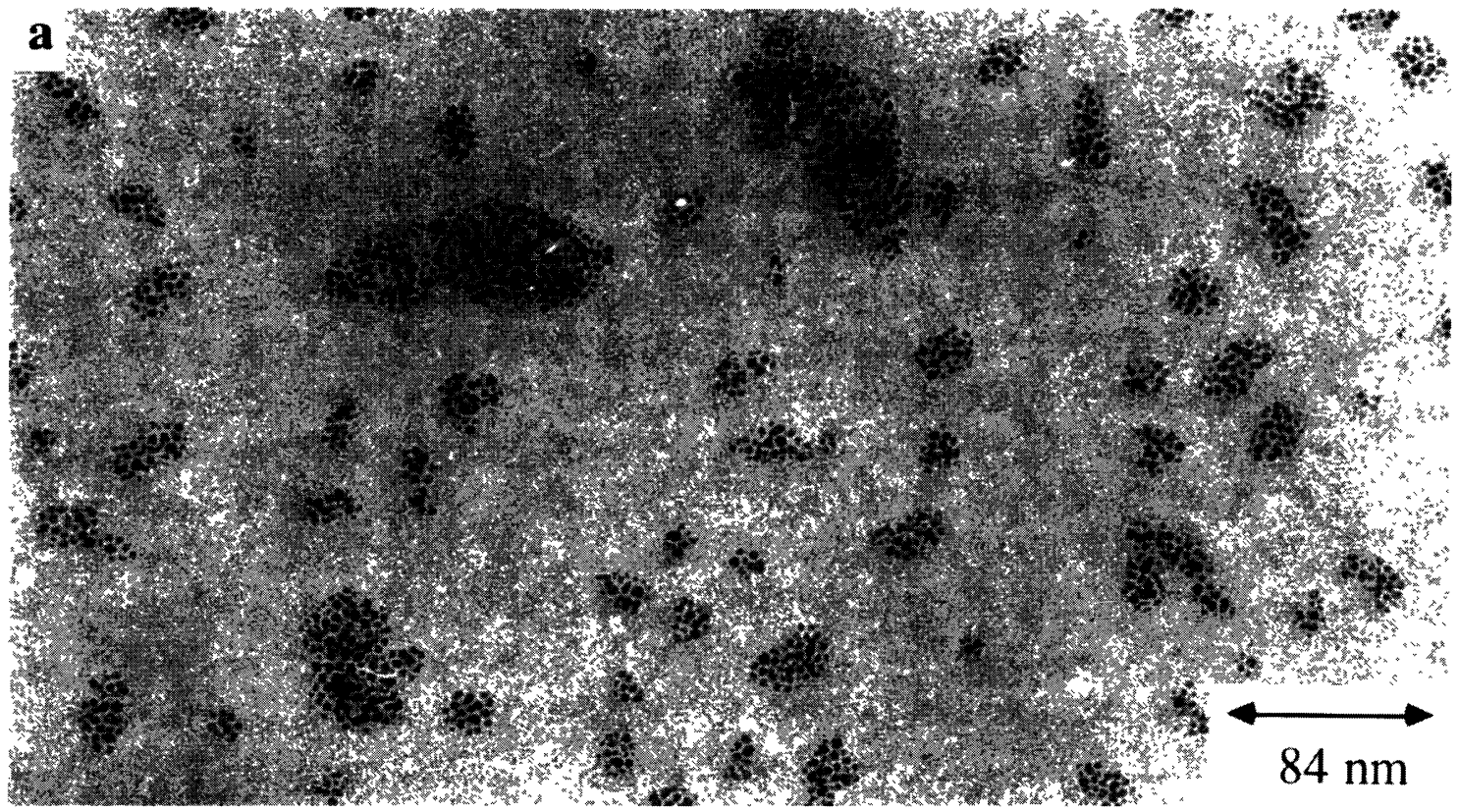

\section{b}
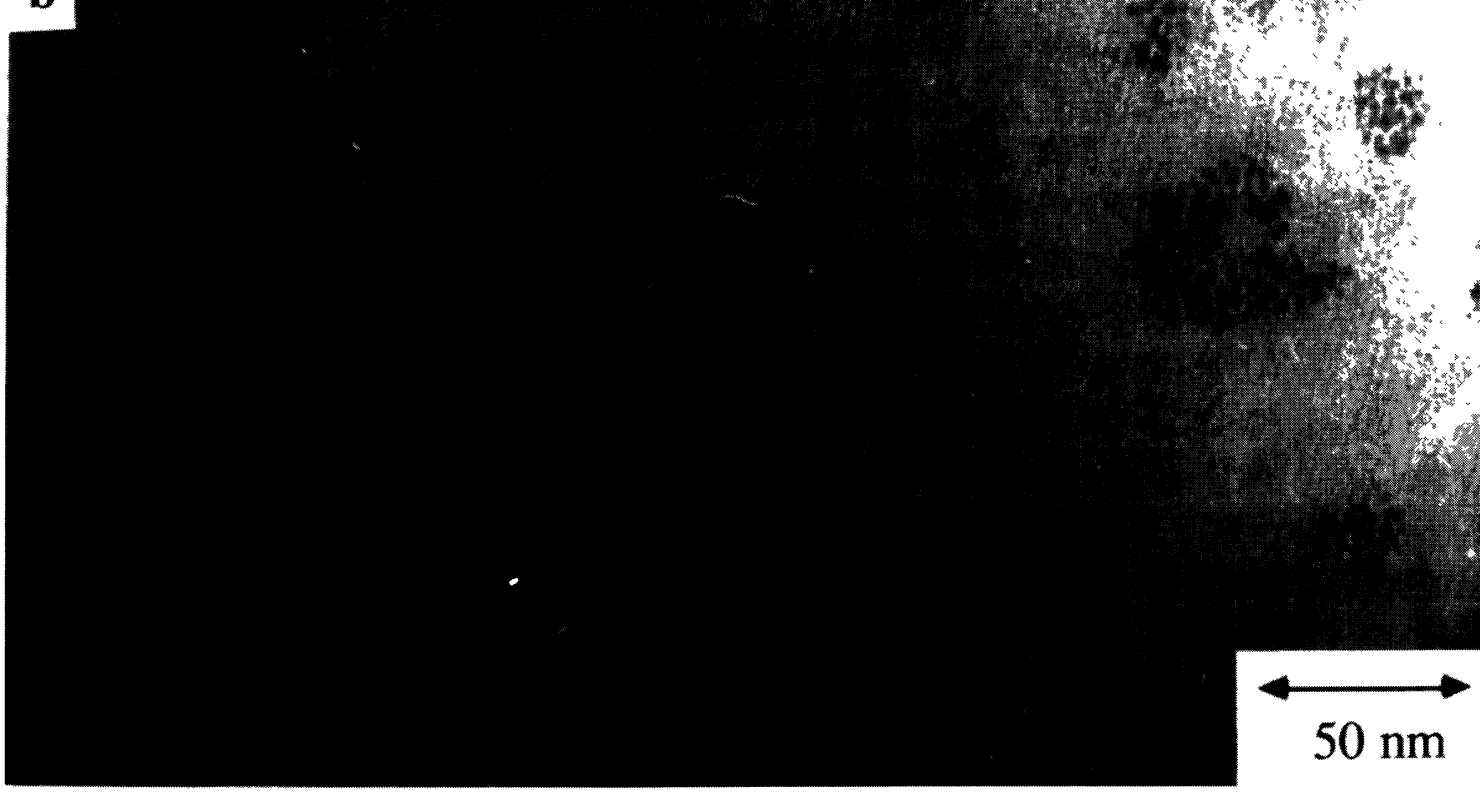

$50 \mathrm{~nm}$

Fig. 1a,b. - TEM micrographs of palladium/HOPG. High voltage is $200 \mathrm{kV}$. 


\section{STM results and discussion.}

4.1 BRIEF COMPARISON OF THE TEM AND STM RESULTS. - Figure 2a shows a film of palladium on HOPG obtained by the classical atomic beam deposition and figure $2 \mathrm{~b}$ shows a similar sample obtained by cluster beam deposition where an isolated group of particles is seen. The white "particles" in figure $2 \mathrm{a}$ are in fact the convolution of a few smaller particles as it was confirmed by scanning smaller areas [13]. The comparison of images presented in figures 2a (ABD) and $2 \mathrm{~b}$ (CBD) clearly suggests a smaller condensation coefficient in the case of CBD (Fig. 2b). This migth be yield by the fact that, as referred in the previous section, the graphite surface is cleaner in the case of $\mathrm{ABD}$ than in the case of CBD. Therefore, in CBD, the presence of impurities on the surface can generate preferential nucleation sites where large amounts of metal nucleate and grow, yielding a much smaller density of particles on the rest of the surface leading to the discrepancy in the condensation coefficient suggested by the comparison of figures $2 \mathrm{a}$ and $2 \mathrm{~b}$ (where fairly localised observations are performed).
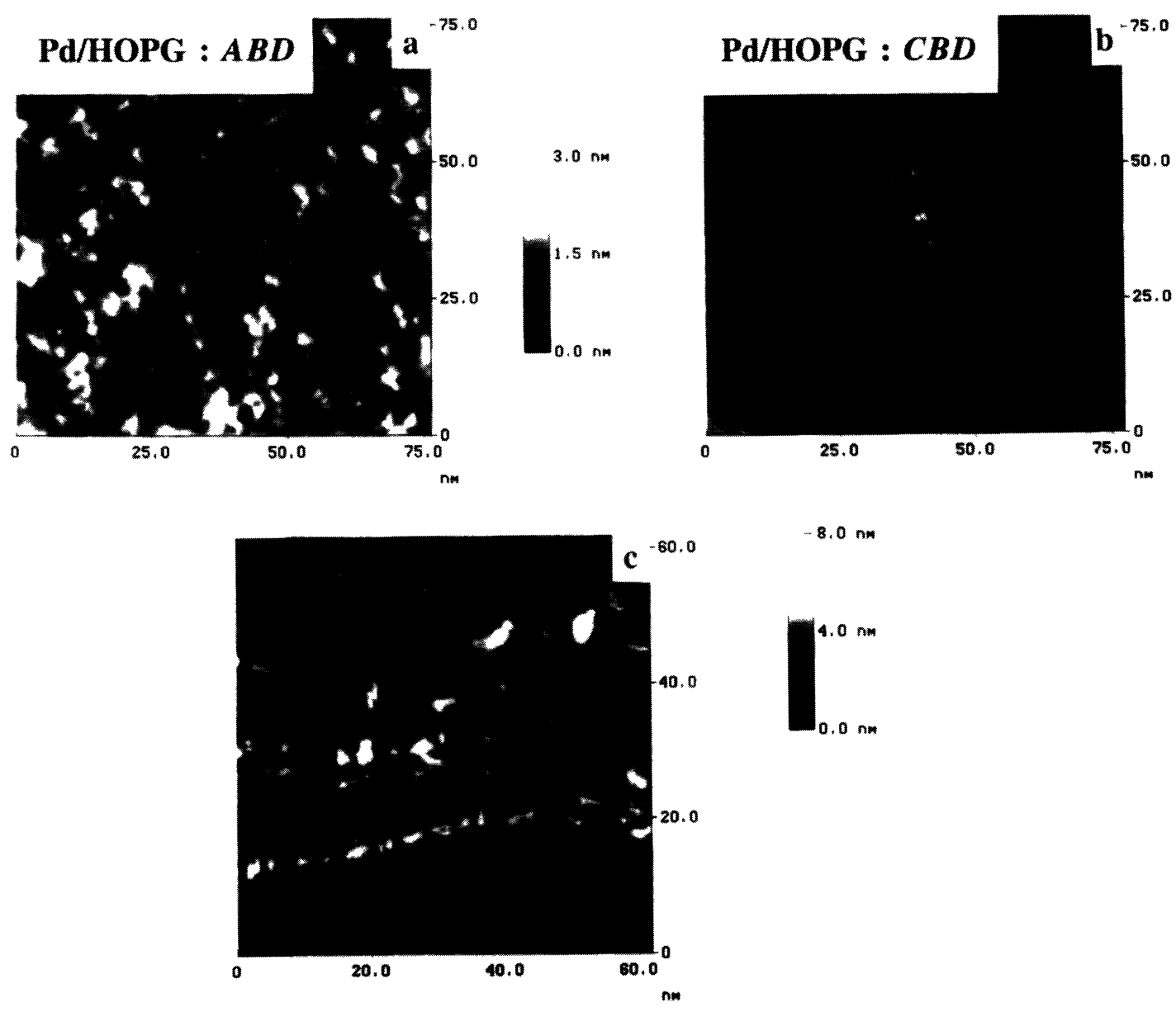

$-8.0 \mathrm{~nm}$

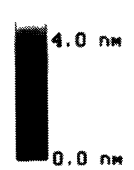

Fig. 2. - STM topographical image. $I=2 \mathrm{nA}, V=50 \mathrm{mV}$. a) Pd/HOPG obtained by atomic beam deposition (ABD); b) Pd/HOPG obtained by cluster beam deposition (CBD); c) Pd/HOPG obtained by cluster beam deposition. Decoration of graphite steps. 
Figure 2c shows the decoration of HOPG steps by palladium particles of variable size. These STM topographic images are in good agreement with the TEM observations previously presented in section 3.

4.2 CHARGE DENSITY MODULATION OF GRAPHITE. - Superstructures on graphite, corresponding to charge density modulations (CDMs) of graphite have been reported $[4,5,14]$. These modulations are observed near "defects" on graphite such as steps, vacancies, supported particles or adsorbed molecules. The observed modulations, often, have a $(\sqrt{3} \times \sqrt{3}) \mathrm{R} 30^{\circ}$ periodicity (if we take the graphite lattice as a reference) and were seen around platinum [5] and palladium particles [4].

Figure 3a presents an atomic resolution image of a group of particles. In between the particles, we recognise the well-known graphite periodic hexagonal lattice with only one bump in each two dimensional unit cell that contains two carbon atoms. The structure parameter is $0.246 \mathrm{~nm}$ and around the particles, and in particular the one pointed by an arrow, a superstructure corresponding to a modulation of this lattice can be observed. In the same way, in figure $3 \mathrm{~b}$ where atomic resolution, near a graphite step decorated with palladium particles, is achieved, we can see in the upper part of the image a superstructure superimposed to the graphite lattice.

To have a better view of these superstructures we have zoomed on the particle pointed by an arrow in figure $3 \mathrm{a}$ (Fig. 4). It is a $4.5 \times 2.5 \mathrm{~nm}$ particle and its height does not exceed $0.5 \mathrm{~nm}$. We notice that there is not a definite atomic arrangement but that atomic size details can be observed within the particle. The superstructures originated by CDMs extend up to a maximum of $2 \mathrm{~nm}$ from the particle. The three regions $(\mathrm{A}, \mathrm{B}, \mathrm{C})$ on the image appearing with different contrasts all present a $(\sqrt{3} \times \sqrt{3}) \mathrm{R} 30^{\circ}$ structure as observed previously [4].

4.3 GRAPHITE. - Sautet and Joachim [15] have used the electron scattering quantum chemical (ESQC) method to analyse the STM image formation of graphite (0001). In graphite, the carbon atoms are arranged in a honeycomb lattice and its Brillouin zone forms an hexagon at the corners of which the Fermi surface is extremely localised, as it is schematically presented in figure 5 . The succession of layers in graphite corresponds to $\mathrm{ABABAB}$... so that only every other atom of the first layer has a neighbour in the second layer. The electronic interaction between atoms of the first and second layers (when they are above each other) increases the probability for graphite electrons to be reflected towards the bulk, and therefore not transmitted through the tunnel gap and so such atoms yield a smaller current and are not seen in the STM images [14 - 16]. Even if we limit ourselves to the other carbon atom, seen in the image, the wave function centered on this atom is degenerate at the Fermi level. The charge density associated to two possible degenerate wave functions is shown in figure 6 (see [15] for a more detailed analysis). These two wave functions present a periodicity of $(\sqrt{3} \times \sqrt{3}) \mathrm{R} 30^{\circ}$ by comparison to the graphite lattice and this comes from the specific components of the Fermi $k$ vector. The STM (calculated) image (Fig. 7) of graphite is the superposition of the two degenerate wave functions (Figs. 6a and b), which, of course, yieds the $(1 \times 1)$ periodicity of graphite. It corresponds well to an experimental image of graphite (Fig. 8). From recently published results [17], we can assume that the difference between the contrasts of figures 7 (ball contrast) and 8 (triangle contrast) is due to the fact that the calculation (Fig. 7) was made with a tetrahedral platinum tip while the for the experiment we do not know the composition of the apex of the tip, but we can assume it to be different from the one used in the calculation.

4.4 INTERPRETATION OF THE CDMS AROUND THE SUPPORTED PALLADIUM PARTICLES. - Mizes and Foster [14] have simulated the effect of a single point and multiple points (dimers with different bonding configurations, and trimers) defects, on the graphite CDM and so on its STM image. It shows that a single point defect and some of the multiple point (depending on the bonding configuration) defects can generate $\mathrm{a}(\sqrt{3} \times \sqrt{3}) \mathrm{R} 30^{\circ}$ periodic CDM. In the case of palladium clusters 

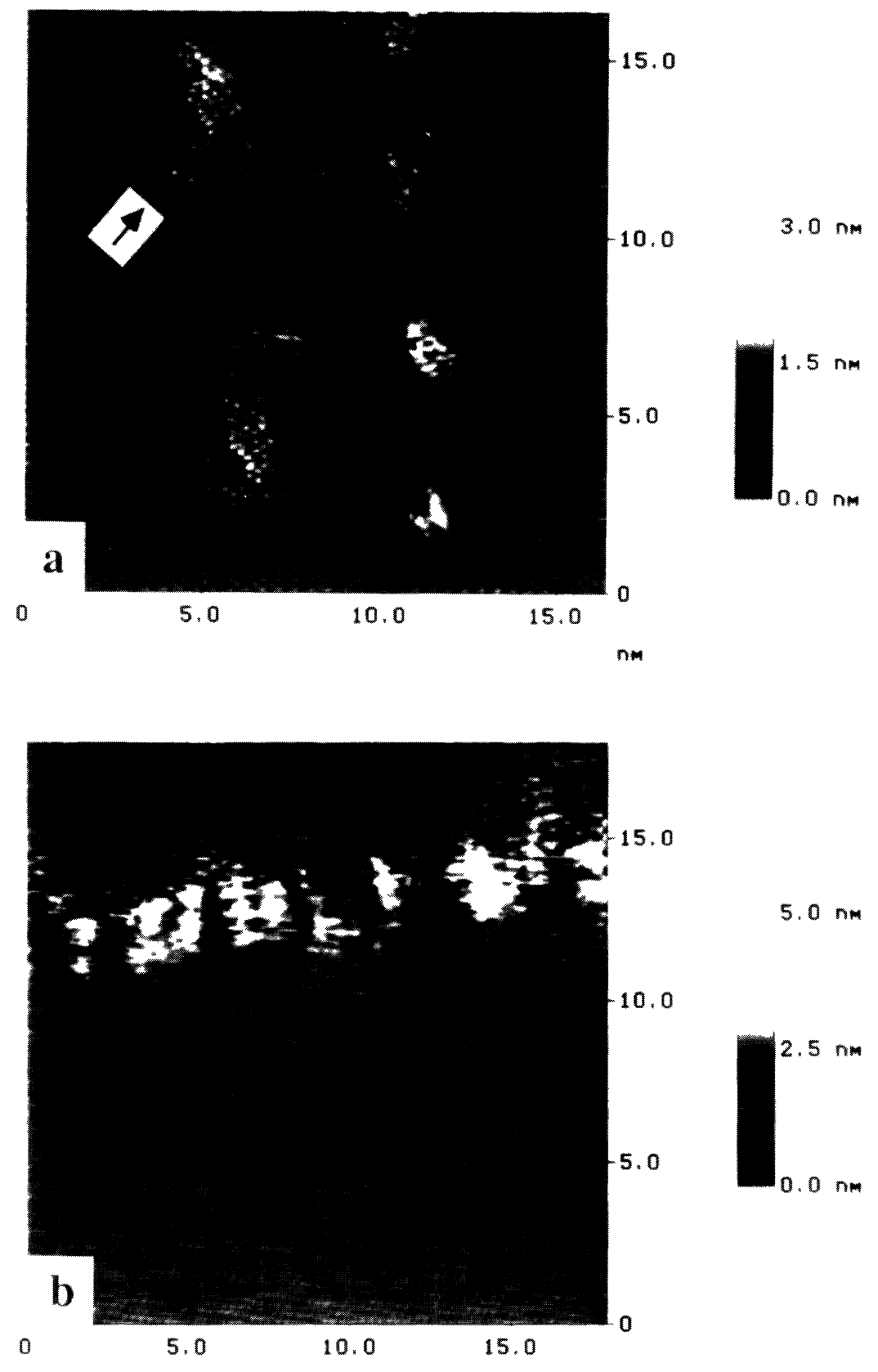

Fig. 3. - STM current image. $I=2 \mathrm{nA}, V=50 \mathrm{mV}$. a) Group of particles (ABD); b) Decorated step (CBD).

we have a large defect compared to an atom. If we take into account the dimensions given above for particle in figure 4 we see that it is formed by about 500 atoms. However, depending on the sites occupied by the surface palladium atoms on the graphite lattice, the influence of the perturbation created by the particle on the graphite charge density can yield an energetic asymmetry on 


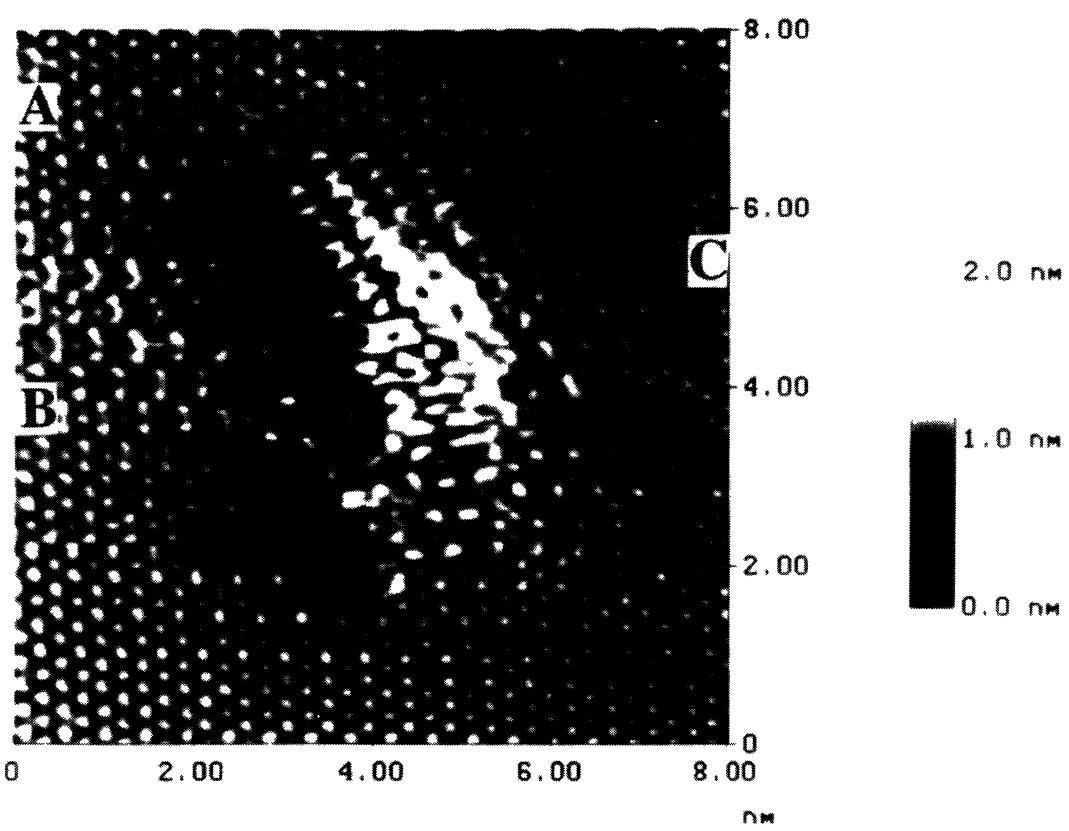

Fig. 4. - STM current image of particle pointed by an arrow in figure $3 \mathrm{a} . I=2 \mathrm{nA}, V=50 \mathrm{mV}$.

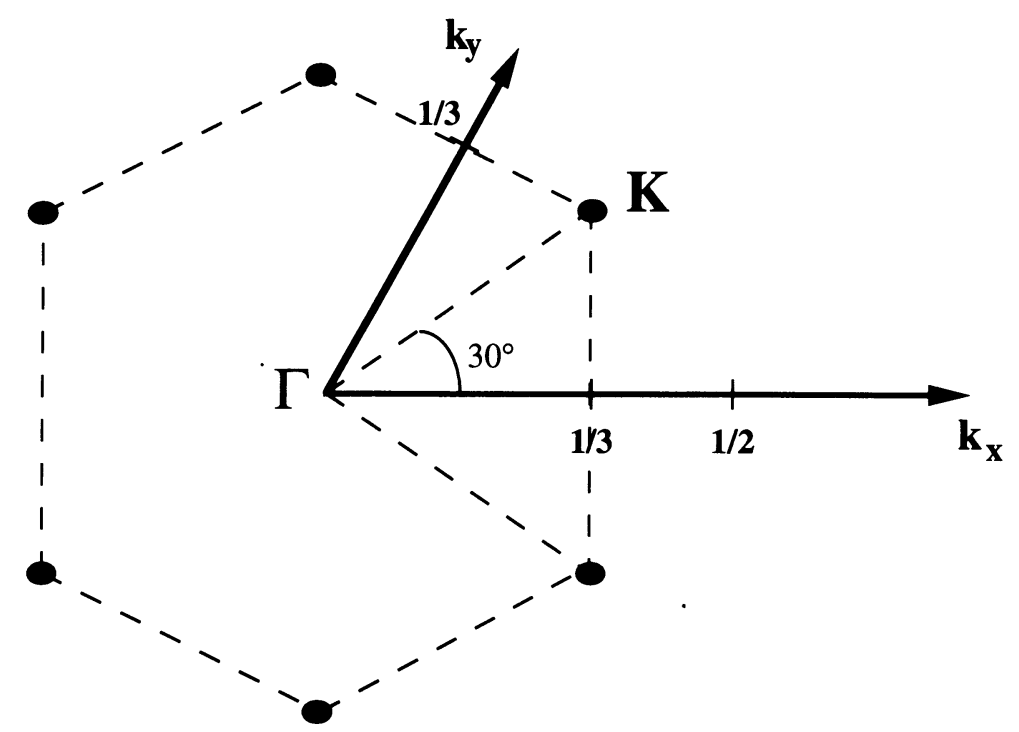

Fig. 5. - Schematic $2 \mathrm{D}$ representation of the Brillouin zone and the Fermi surface of graphite. Axis are normalised to $2 \pi / a(a=0.145 \mathrm{~nm})$. 

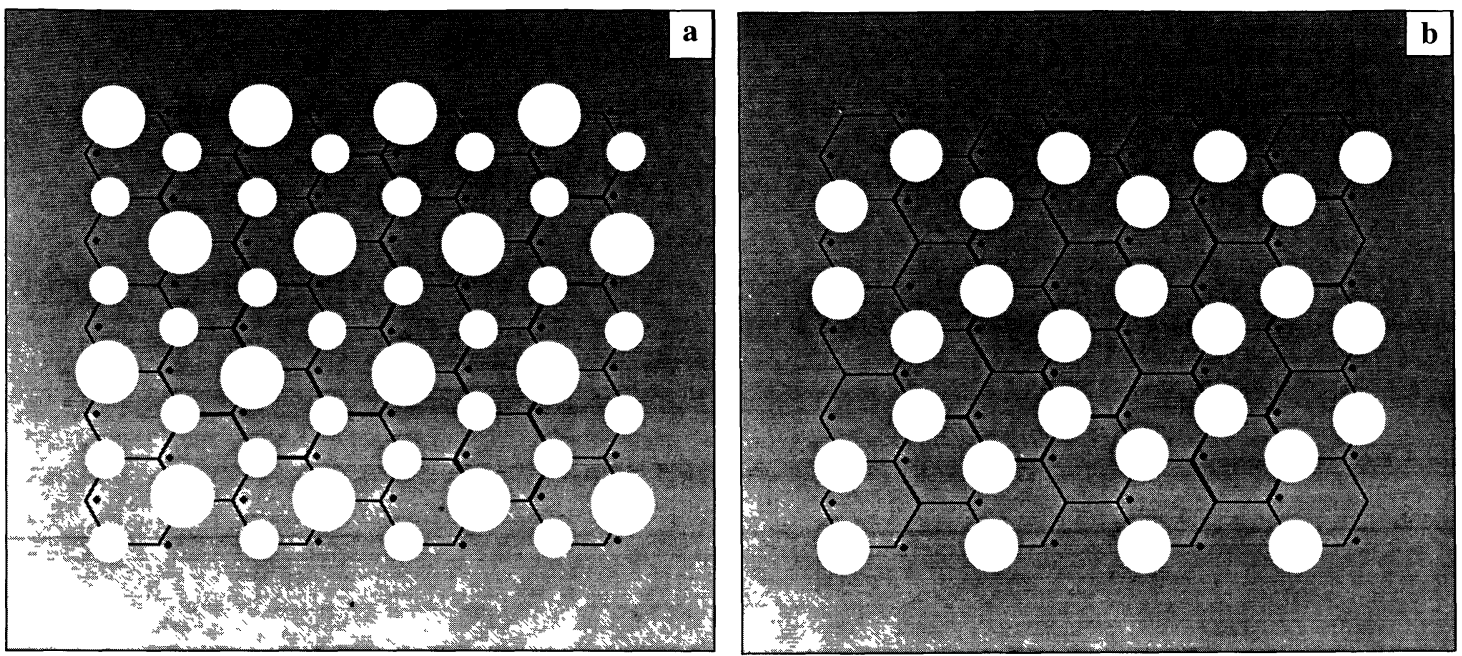

Fig. 6a,b. - Schematic representation of the two degenerated wave functions of graphite at the Fermi energy level. The atoms with a neighbour in the second layer are indicated.

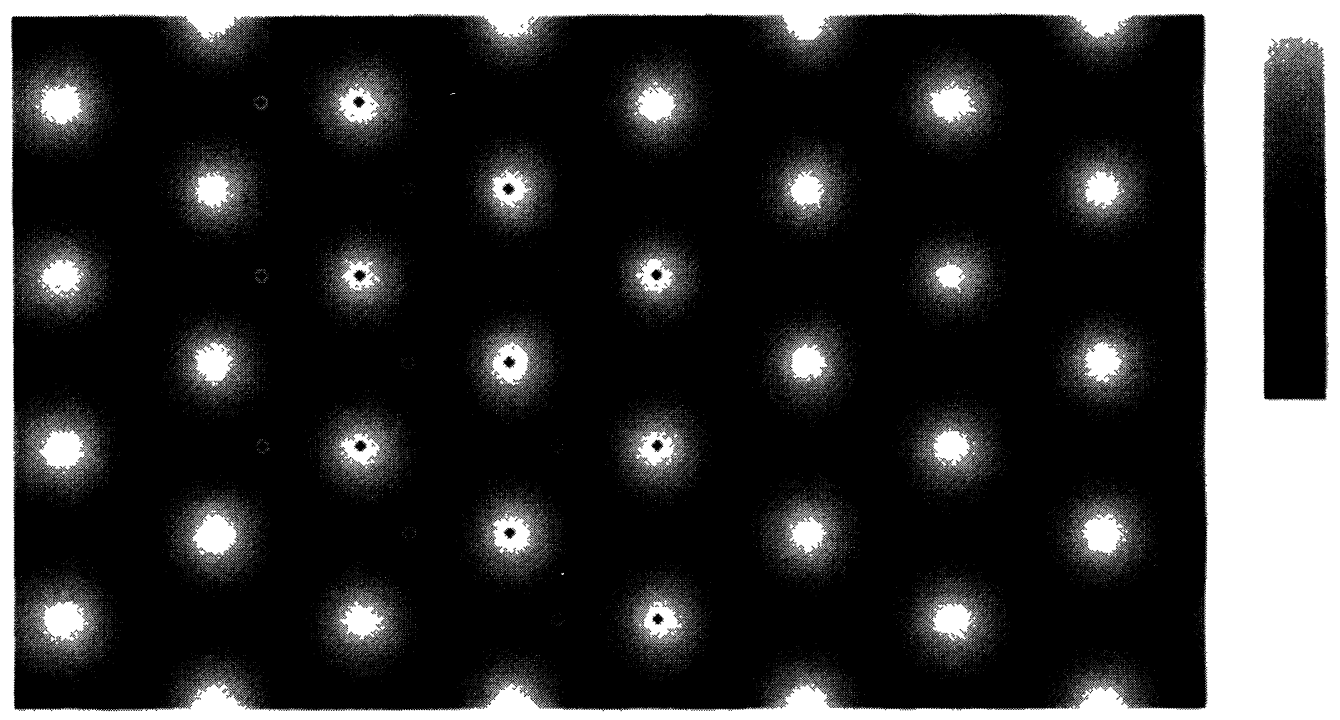

Fig. 7. - Calculated image of graphite (0001). $I=2 \mathrm{nA}, V=20 \mathrm{mV}$. The calculation was made on the 18 carbons of the first layer that are indicated. Scale is from $0.365 \mathrm{~nm}$ (black) to $0.446 \mathrm{~nm}$ (white).

the two degenerate wave functions (see Figs. 6a and b) and so the degeneracy is removed. At the Fermi level, one of the wave functions, participating to the image formation of graphite, will be strengthened while the other will be weakened, resulting in a $(\sqrt{3} \times \sqrt{3}) \mathrm{R} 30^{\circ} \mathrm{CDM}$.

If we now have a close look at the three regions (A, B, C) of figure 4 (Fig. 9a, b and c). In the upper part of figure $9 \mathrm{a}$ we recognise the graphite lattice and within it, in the rhombus formed by four carbon atoms along the horizontal axis, we notice that the two "black" (low-density) sites are 


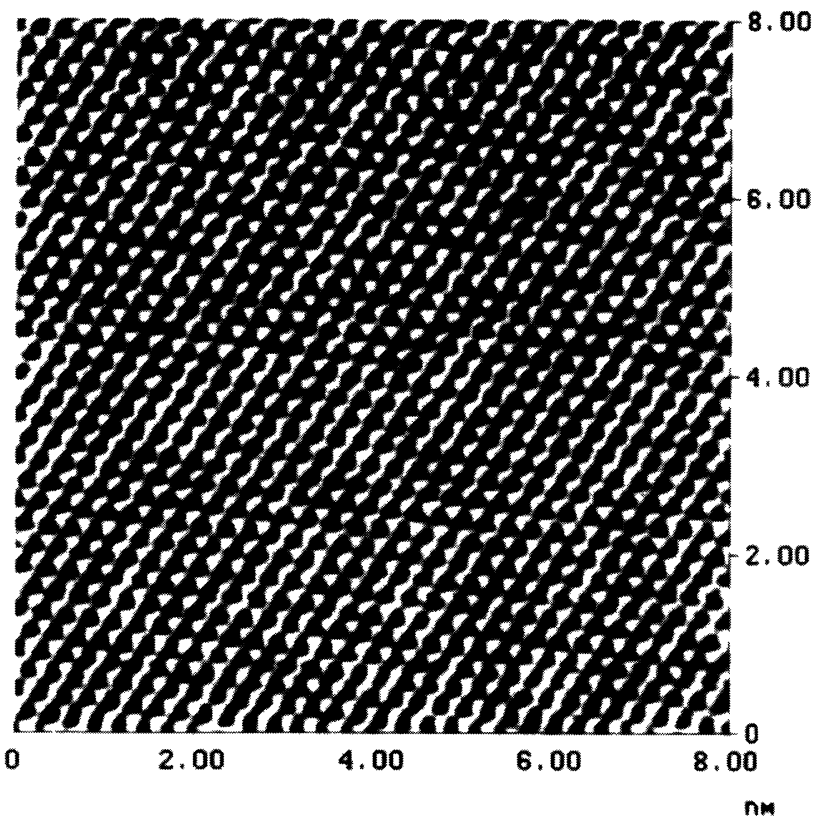

8.00

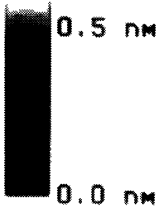

\section{HOPG}

Fig. 8. - Experimental STM image of graphite. $I=2 \mathrm{nA}, V=50 \mathrm{mV}$.

not equivalent, since one is "black" and the other "grey". From the calculations, the "grey" site corresponds to the place of the atom which has a neighbour in the second layer, while the "black" site corresponds to a hollow. Near the particle border (middle of Fig. 9a) we notice a "clover" like features within the $(\sqrt{3} \times \sqrt{3}) \mathrm{R} 30^{\circ}$ periodic CDM, meaning that the previously called "grey" site is now equivalent (in terms of charge density) to the hollow ("black") site. In the bottom of the same figure we can see that the contrary happens and the charge density of the so-called "grey" site strongly increases becoming similar to the charge density of the sites forming the $(\sqrt{3} \times \sqrt{3}) \mathrm{R} 30^{\circ}$ periodic CDM. Region B (Fig. 9b) and region C (Fig. 9c) can be considered similar to the bottom of region A. It seems reasonable to adventure the hypothesis that, depending on the region of the particle, the surface atoms of palladium that interact with the graphite are not placed at equivalent sites, giving rise to different contrasts within the periodic CDM, originated by the fact that the perturbation lifts up the degeneracy of the two wave functions of graphite at the Fermi energy level. The fact that we have a different interaction of the degenerate wave functions with such a large particle means that the interaction between the particle and the substrate is not random but presents a short range order with a $(\sqrt{3} \times \sqrt{3})$ type epitaxy. Since the CDMs are different from place to place around the particle, there is no long range order of the possible $(\sqrt{3} \times \sqrt{3})$ domains.

Another point that deserves a comment is the fact that the CDMs around palladium particle extend up to a maximum of $2 \mathrm{~nm}$ from the particle, while in the case of platinum particles these can extend up to $5 \mathrm{~nm}$. The key to the explanation of this difference is, certainly, related to the fact that the $d$ band in palladium is narrower than in platinum. The width of the $d$ band of palladium is roughly the half of the $d$ band width of platinum [18]. The interaction between the $\mathrm{p}$ electrons of graphite and the $d$ band of the metal is then expected to be active to a larger extent for the platinum than for palladium.

Finally, on the flatness of the particles observed by STM (thickness $\leq 0.5 \mathrm{~nm}$ ), it can be said that, at room temperature, growth is mainly two dimensional, but also, that the STM image of 

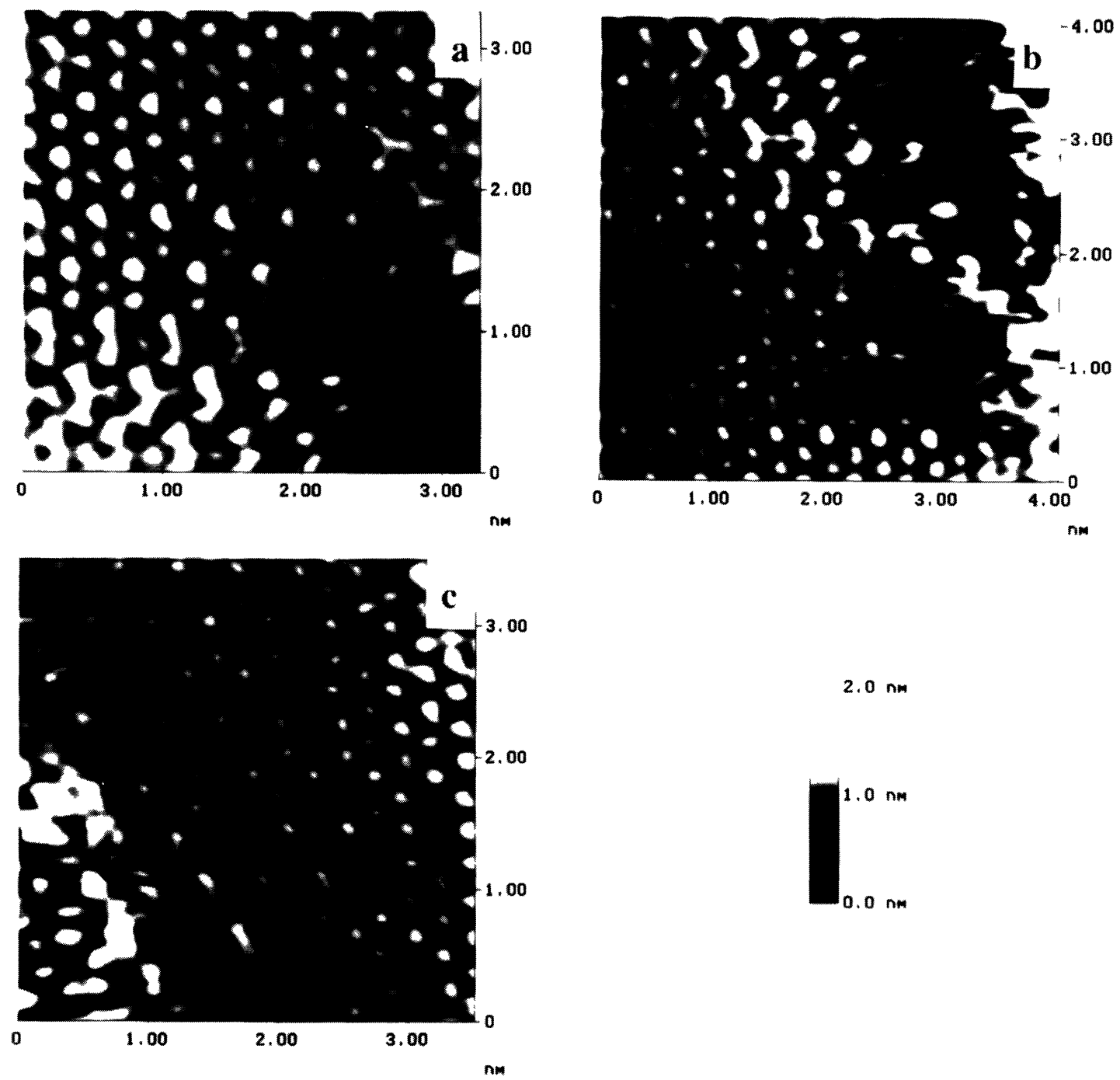

$2.0 \mathrm{~nm}$

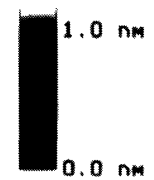

Fig. 9. - Regions A (a), B (b) and C (c) of figure 4.

these particles are certainly the fingerprint of the electronic interaction of the palladium particle with graphite and not a direct image of the particle in three dimensions.

\section{Conclusion.}

We have characterised by TEM and STM a palladium film obtained by cluster beam deposition. TEM and STM images agree to show a great difference in density of the supported particles and their mean size (by comparison to the atomic beam deposition films). In order to perform an efficient correlation between the physical characteristics of the particles and their catalytic properties as a function of their size, one must go now a step further and do a mass selection on the size distribution in order to narrow the size distribution of the supported particles. We must, 
however keep in mind that even large particles are mobile on the substrate and that it will be difficult to have a monodisperse collection of supported particles.

The $(\sqrt{3} \times \sqrt{3}) \mathrm{R} 30^{\circ}$ periodic CDMs observed around the particles are interpreted in the terms of a perturbation (particle) that lifts up the degeneracy of the wave functions of graphite at the Fermi energy. The interpretation is not as simple as with a single point perturbation but one can assume that the contrast variations observed within the image can be attributed to different site occupation, on the graphite lattice, of the atoms at the border of the palladium particle.

Along the decorated graphite steps another kind of CDMs were observed but not presented, namely $(\sqrt{3} \times \sqrt{3}) \mathrm{R} 30^{\circ}$, that may have their origin on the orientation of the step. This will make the object of a future paper, since their origin is not yet fully understood.

The study of the interactions of the particles with the substrate are very useful in the understanding of the catalytic behaviour of small supported particles.

\section{Acknowledgements.}

Professor Tran Minh Duc is greatly thanked for giving access to the CENATS scanning tunneling microscopy facilities. We are equally grateful to Dr. Bernadette Tardy for providing the atomic beam deposition sample, and to Mr. G. Guiraud for technical assistance. The cluster generator used in this work is part of the Centre Interlaboratoires de Recherches sur les Petits Agrégats (Villeurbanne) which is administered by three laboratories connected with the CNRS (IRC - UPR 5401; LASIM -URA 171; DPM - URA 172).

\section{References}

[1] Humbert A., Dayez M., Sangay S., Chapon C. and Henry C.R., J. Vac. Sci. Technol. A8 (1990) 311.

[2] Humbert A., Dayez M., Granjeaud S., RicCi P., Chapon C. and Henry C.R., J. Vac. Sci. Technol. B9 (1991) 804.

[3] SATTLER K., Z. Phys. D (1991) 287.

[4] Porte L., Phaner M., Noupa C., TARdy B. and Bertolini J.C., Ultramicroscopy 42-44 (1992) 1356.

[5] Xhie J., Sattler K., Muller U., Venkateswaran N. and Raina G., Phys. Rev. B43 (1991) 8917.

[6] Sartre A., Phaner M., Porte L. and Sauvion G.N., Preprint.

[7] SMALLEY R.E., Laser Chem. 2 (1983) 167.

[8] CADETE SANTOS AIRES F.J., PhD Thesis (1990), University of Lyon (unpublished).

[9] Fuchs G., TreilleuX M., Santos Aires F., Cabaud B., Melinon P. and Hoareau A., Phys. Rev. A40 (1989) 6128.

[10] Fuchs G., Melinon P., Santos Aires F., Treilleux M., Cabaud B. and Hoareau A., Phys. Rev. B44 (1991) 3926.

[11] Tardy B., Noupa C., Leclerco C., Bertolini J.C., HoAreau A., TreilleuX M., Faure J.P. and NiHOUL G., J. Catal. 129 (1991) 1.

[12] Melinon P., Cabaud B., Hoareau A., Jensen P., Paillard V. and Treilleux M., J. Phys. I France 3 (1993) 1585.

[13] CADETE SANTOS AIRES F.J., unpublished.

[14] MIZES H.A. and FOSTER J.S., Science 244 (1989) 559.

[15] SAUTET P. and JOACHIM C., Ultramicroscopy 42-44 (1991) 115.

[16] TomaneK D., Louie S.G., Mamin H.J., Abraham D.W., Thomson R.E., Ganz E. and Clarke J., Phys. Rev. B35 (1987) 7790.

[17] DunPHY J.C., OGLETREe D.F., SAlmeron M.B., SAUTET P., BOCQueT M.L. and JOACHIM C., Ultramicroscopy $42-44$ (1992) 490.

[18] SAUTET P. and PAUL J.F., Catalysis Lett. 9 (1991) 245. 